\title{
Kinematic GNSS positioning results compared against Agisoft Metashape and Pix4dmapper results produced in the San Joaquin experimental range in Fresno County, California
}

DOI: https://doi.org/10.1515/jogs-2020-0122

Received September 17, 2020; accepted July 13, 2021

\begin{abstract}
RTKLIB which is an open source Global Navigation Satellite Systems (GNSS) software has gained rapid acceptance among Surveying professionals thanks to recent developments in UAS (Unmanned Aerial System) technology. RTKLIB enables standard and precise point positioning (PPP) in real-time and post-processing modes to be performed. As such, UAS users utilize this software to analyze GNSS data collected by GNSS systems on UAS. By being versatile and free, RTKLIB is commonly used by many; however, it is not the only freely available GNSS software. There are also freely available online GNSS data processing software running on servers. These online GNSS data processing services provide data processing in static, kinematic and rapid static modes. Because UAS collect data in kinematic mode, in this study, kinematic data processing by aforementioned software (CSRS-PPP, GAPS and APPS) is analyzed. The results coming from these software are compared against the results produced by photogrammetric software (Agisoft Metashape and Pix4Dmapper). The aim of this practical project is to produce generalizable knowledge about the performance of these software. It is found out that RTKLIB and CSRS-PPP achieved cm-level precision. Yet, GAPS and APPS achieved dm-level precision both for horizontal and vertical coordinates. This study demonstrates the precision and accuracy expected from these software if they are used for kinematic GNSS data processing.
\end{abstract}

Keywords: Agisoft Metashape, Online GNSS Data Processing, Photogrammetry, Pix4Dmapper, RTKLIB

\footnotetext{
*Corresponding Author: M. Berber: Department of Civil and Geomatics Engineering, California State University, Fresno, CA, E-mail: muberber@csufresno.edu

R. Munjy, J. Lopez: Department of Civil and Geomatics Engineering, California State University, Fresno, CA
}

\section{Introduction}

UAS (Unmanned Aerial System) photogrammetry is a measurement tool of surveying that has grown widely in the last ten years. It combines traditional photogrammetry techniques with structure from motion (SfM). SfM is widely used because it provides greater flexibility and high quality results (Chiabrando et al., 2013). SfM is a computer vision technique which uses image overlaps, camera position (and orientation if available) to solve for the geometry of the scene, camera interior and exterior parameters without providing known 3D point positions (AgüeraVega et al., 2017). UAS often used for photogrammetry are equipped with survey-grade, dual frequency Global Navigation Satellite Systems (GNSS) receivers that report a priori standard deviations of 1 to $2 \mathrm{~cm}$ horizontally and 3 $\mathrm{cm}$ vertically using differential GNSS with a base station. Some UAS are equipped with inertial navigation systems that record 3D rotation angles of the aircraft body at the instant of exposure; however, body rotation measurements are primarily used for navigation. The aerotriangulation process of aerial photogrammetry uses initial estimates of exterior and interior camera orientation provided by the combination of onboard sensors and surveyed ground control information to compute the position and orientation of the camera at the time of exposure. By constraining the ground control, the camera positions are computed using the bundle adjustment (Fraser, 1997; Pérez et al., 2011; Nocerino et al., 2013; Kemper et al., 2016 and Tekavec et al., 2019). The bundle solution outputs the camera perspective center exterior orientation. GNSS positioning outputs the UAS antenna position. The difference between the two solutions is a bias usually in the $\mathrm{z}$ direction that reflects the lever arm of the GNSS antenna. In this study, the results coming from the bundle adjustment were used for the baseline coordinates.

A portion of this manuscript investigated the current status of UAS (particularly referring to Small Unmanned 
Aerial Systems, sUAS) photogrammetric mapping technology using digital cameras and Light Detection and Ranging (LIDAR) and provided operational specifications, including minimums, for utilizing UAS through a comparative analysis of data obtained with UAS to data obtained from traditional ground surveying methods. Such a systematic comparative analysis is designed to identify the relative strengths and drawbacks of this new technology and where it can be effectively used for mapping projects. As such, this research project brought the latest technology into the photogrammetric arena. GNSS portion of this project analyzed the data collected utilizing these emerging technologies using RTKLIB and three online kinematic GNSS data processing services.

RTKLIB is a commonly used open source program package for GNSS positioning. This program package has been developed by Tomoji Takasu since 2006. Currently, the latest stable version of RTKLIB is 2.4 .2 p13. RTKLIB consists of a portable program library and several Application Programs Interfaces (API's) utilizing the library. The features of RTKLIB (http://www.RTKLIB.com) include supporting standard and precise positioning with GPS, GLONASS, Galileo, QZSS, BeiDou and SBAS. It supports various positioning modes with GNSS for both realtime and post-processing: Single, DGPS/DGNSS, Kinematic, Static, Moving-Baseline, Fixed, PPP-Kinematic, PPP-Static, and PPP-Fixed. One advantage of using RTKLIB is that all of the executable binary API's for Windows are included in the package as well as whole source programs of the library and the API's.

Currently, three open-source services provide online kinematic GNSS data processing: Auutomatic Precise Positioning Service (APPS), Canadian Spatial Reference System Precise Point Positioning Service (CSRS-PPP) and GPS Analysis and Positioning Software (GAPS). Online GNSS data processing services have been studied by many authors for more than a decade see for instance Soler et al. (2006), Ghoddousi-Fard and Dare (2006), Liu and Shih (2007), Tsakiri (2008), Ebner and Featherstone (2008), Snay et al. (2011a, b and c), Berber et al. (2014), Berber and Wright (2016), Berber et al. (2017), Jamieson and Gillins (2018) and Dogru et al. (2018). Since online GNSS data processing will not be further explained in this paper, interested readers are referred to these publications for detailed information on the use of these services. Aforementioned references mostly investigated static GNSS data processing using online services. In this study, kinematic data collected by a GNSS receiver on an UAS is analyzed using RTKLIB and three online kinematic GNSS data processing services. In kinematic mode, usually a few seconds to a few minutes data is collected. With GNSS data analysis, the rule of thumb is that the longer the observation period at a location, the more precise the results will be. Since in this study GNSS data is collected by a receiver by UAS, the observation period is tenth of a second, i.e., shorter than the observation period commonly used for kinematic surveys (Ghilani and Wolf (2007)).

In this manuscript, 81 control points located in the San Joaquin Experimental Range in Fresno County, California were utilized for a UAS photogrammetry campaign. DJI Inspire 2 Pro model aircraft was chosen for the aircraft platform. The collected data was processed using Agisoft Metashape and Pix4Dmapper. In the end, a bundle block adjustment was performed applying camera selfcalibration. The result of the photogrammetric bundle adjustment were considered the baseline and differences between the results produced by RTKLIB, APPS, CSRS-PPP and GAPS software and the bundle adjustment were computed.

\section{Methods}

A UAS photogrammetry campaign was flown over the San Joaquin Experimental Range located in Fresno County, California in the foothills of the Sierra Nevada Mountain Range located about $32 \mathrm{~km}$ north of the California State University, Fresno campus. There were 81 control points (Fig. 1) laid out in a 9-by-9 grid spaced approximately $40 \mathrm{~m}$ apart throughout a $320 \mathrm{~m}$ by $320 \mathrm{~m}$ area. The control point flight targets were designed to be circular, black and white, and measured about $47 \mathrm{~cm}$ in diameter (Fig. 2). These control points were surveyed to $1 \mathrm{~cm}$ horizontal and $0.3 \mathrm{~cm}$ vertical accuracy both at one sigma confidence level. The horizontal positioning was established using static GNSS at two different times on February 10-17, 2018 and July 1218, 2018. For this purpose, six Trimble-R8 Model-2 receivers are employed to collect the measurements. Antenna and receiver are mounted on a fixed-height 2-m range pole. Each point is occupied minimum 30 min with $1 \mathrm{~s}$ interval; however, while moving from point to point, some stations collected longer data. To determine the elevations of the points, differential levelling was utilized using the Trimble DiNi digital level with an adjustable level rod. Local vertical control was surveyed in from a National Geodetic Survey (NGS) benchmark west of the project site just north of the intersection of Highway 41 and Road 406. After establishment of the project vertical benchmark (point 22), the remaining 80 control locations were then measured. A vertical network was created with a total 154 observations to establish the elevations of each control point. The 
difference in elevation along each link between each individual control point was measured. Interior control points were measured with a minimum of 4 observations, exterior control points were measured with a minimum of 3 observations, and the corners of the site were measured with a minimum of 2 observations. All of these measurements were input into STAR ${ }^{\star} \mathrm{NET}$ for a simultaneous least squares adjustment.

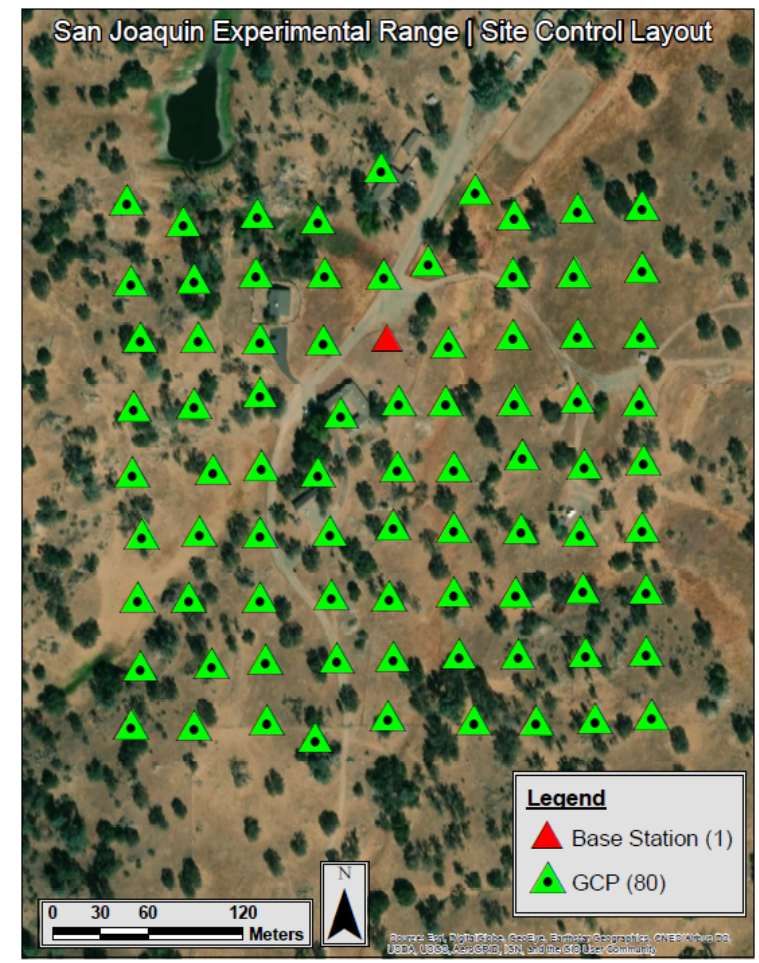

Fig. 1. Control points established in the project site (image is prepared using ESRI ArcMap).

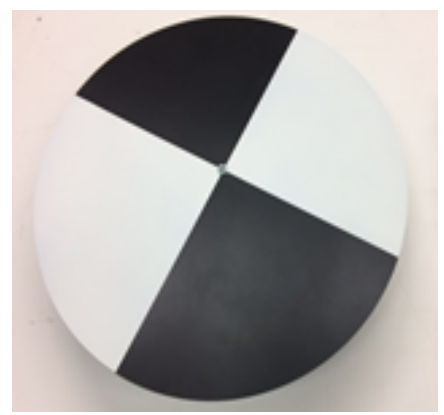

Fig. 2. Ground target image.

The aircraft platform used was a DJI Inspire 2 Pro model aircraft, a vertical-take-off-and-landing platform with a quad rotor. The system included Loki, a third-party dual-frequency GNSS receiver. The Loki receiver was triggered by the hot shoe flash, which approximated the midexposure pulse. The camera model was FC6510, with a focal length of $8.8 \mathrm{~mm}$, pixel size of $0.0024 \mathrm{~mm}, 5472$ pixels wide, 3648 pixels in height and the number of photos was 837 for the entire flight. This entire flight included two lifts: lift 1 and lift 2 . In this study, data from lift 1 was used. The flight line pattern can be seen in Fig. 3 .

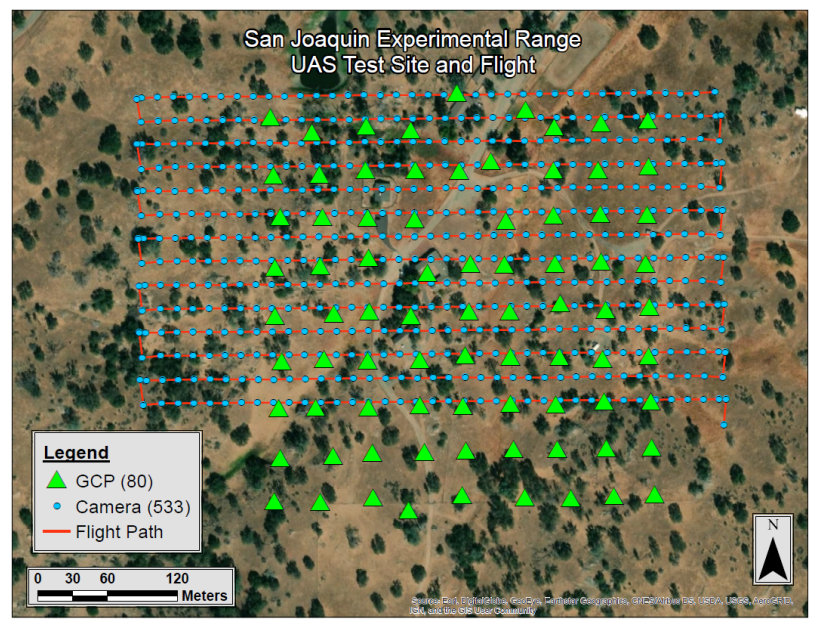

Fig. 3. Flight trajectory image for the project site.

The flight plan had an average flying height of $75 \mathrm{~m}$ above ground level, and forward and side overlap percentage both of $80 \%$. The images were processed using Agisoft Metashape and Pix4Dmapper, two popular commercial software for processing UAS imagery. The images were added to a software project and matched and aligned to create the initial block to be adjusted according to the surveyed ground control. Camera positions in the bundle adjustment were assigned an a priori uncertainty of $1.0 \mathrm{~m}$ both horizontally and vertically. Next, 80 control points (one of the 81 control points was occupied as a base station during the flight and therefore was not included in the final bundle block adjustment) were added to the project and measured with an uncertainty of 0.5 pixels in all images in which they appeared. The ground control points were all constrained by uniformly assigning a priori uncertainties of $1.0 \mathrm{~cm}$ horizontally and $0.5 \mathrm{~cm}$ vertically according to the accuracy of the survey. Finally, a bundle block adjustment was performed applying camera self-calibration (focal length, principal point, and lens distortion parameters).

For this flight, the GNSS base station was established on control point number 22 (in figure 1, point 22 was lo- 
cated in the space, from the left, in the $3^{\text {rd }}$ row, $5^{\text {th }}$ column) and data was collected over a duration of two hours. At the end of the flight two RINEX files were generated, one for the base and one for the rover on the UAS. These two files along with the navigation data file were fed into RTKLIB, CSRS-PPP, GAPS and APPS software to determine the camera position coordinates which were taken during the aerial survey.

RTKLIB (http://www.RTKLIB.com) requires above mentioned two RINEX files along with the navigation data file. Figure 4 displays the data processing options used in RTKLIB.

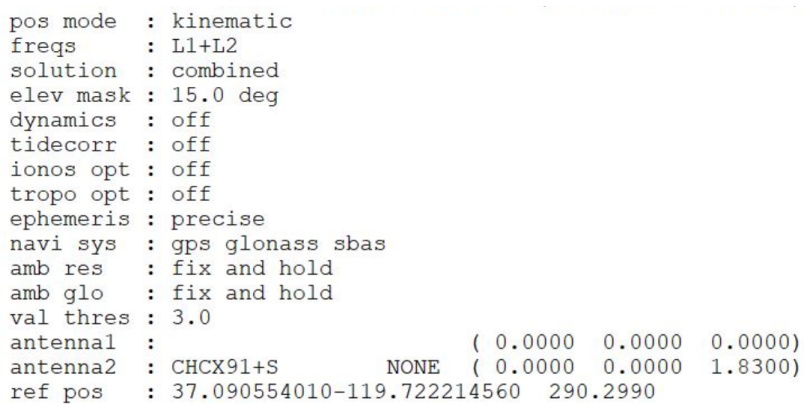

Fig. 4. Data processing parameters used in RTKLIB.

Utilizing PPP technique, APPS (http://apps.gdgps. net/) requires only the rover data because it accesses precise ephemeris and clock parameters from the servers such as International GNSS Service (IGS) server. Figure 5 shows the processing options entered in APPS.

\begin{tabular}{|l|l|}
\hline \multicolumn{2}{|c|}{ APPS Options } \\
\hline Processing Mode & $\begin{array}{l}\text { O Static } \\
\text { Kinematic }\end{array}$ \\
\hline Measurement Type & $\begin{array}{l}\text { Single Frequency } \\
\text { Dual Frequency }\end{array}$ \\
\hline Orbits/Clocks used & $\begin{array}{l}\text { JPL Final: Data prior to 2020-07-04 } \\
\text { JPL Rapid: Data from 2020-07-04 to 2020-07-11 } \\
\text { JPL Ultra R/T: Data from 2020-07-11 to present }\end{array}$ \\
\hline L1 Code & $\begin{array}{l}\text { OCA Code } \\
\text { OP Code }\end{array}$ \\
\hline Model Pressure Data? & Yes O No \\
\hline $\begin{array}{l}\text { Elevation Dependent } \\
\text { Data Weighting }\end{array}$ & OFlat $\bigcirc$ Sin $\bigcirc$ Sqrt(sin) \\
\hline Advanced Options & $7.5 \quad$ Elevation Angle Cutoff \\
\hline
\end{tabular}

Fig. 5. Data processing parameters used in APPS.
As being a PPP technique, CSRS-PPP (http://webapp. geod.nrcan.gc.ca/geod/tools-outils/ppp.php) only needs the rover data as well (see Figure 6).

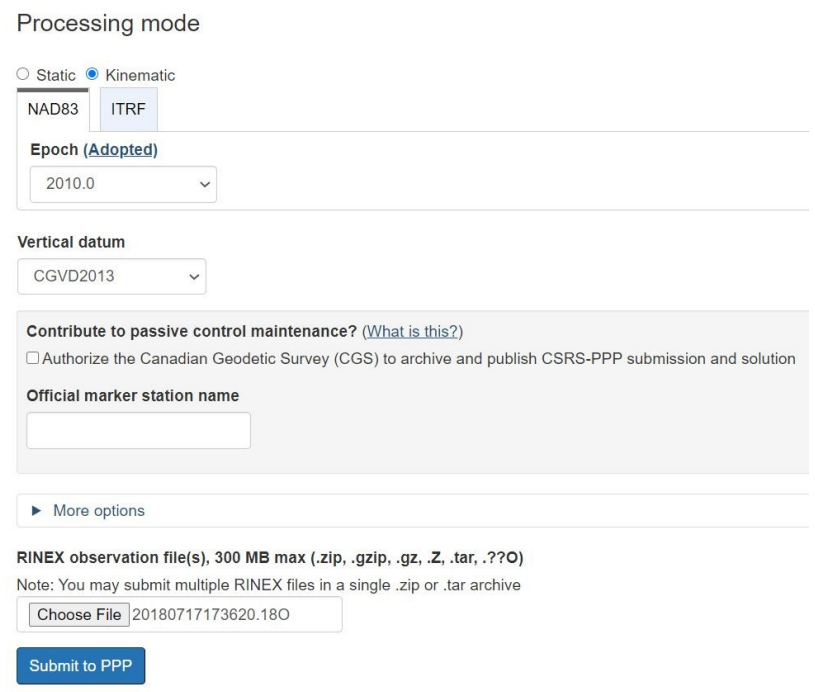

Fig. 6. Data processing parameters used in CSRS-PPP.

The same rover file is also submitted to GAPS (http: //gaps.gge.unb.ca/), see Fig. 7.

The results produced by these services/software are compared against aerial triangulation results and discrepancies are calculated.

\section{Application and Results}

In this project, after setting up the base station, kinematic GNSS data is collected using DJI Inspire 2 Pro model aircraft. At the end of the flight, two RINEX files were generated, one for the base and one for the rover on the UAS. As needed, by submitting these files into RTKLIB, CSRS-PPP, GAPS and APPS software, the camera position coordinates are determined. Camera position coordinates were also determined using aerial triangulation via Agisoft Metashape and Pix4Dmapper software. In this project, aerial triangulation coordinates were considered the baseline and differences between the results produced by RTKLIB, CSRSPPP, GAPS, APPS software and aerial triangulation methods were computed. The discrepancies between the results produced by these software and Agisoft and Pix4D are shown in Figs. 8-11 and Figs. 12-15, respectively. All the coordinates are produced in NAD83 (2011) (Epoch 2010), and ellipsoidal height was utilized for elevations. 
Table 1. Results about latitude differences (Agisoft) (m).

\begin{tabular}{ccccc}
\hline & RTKLIB & CSRS-PPP & GAPS & APPS \\
\hline Min & -0.216 & 0.194 & -0.342 & -1.329 \\
Max & 0.040 & 0.446 & 0.749 & -0.223 \\
Mean & -0.049 & 0.361 & 0.466 & -0.722 \\
Range & 0.255 & 0.252 & 1.092 & 1.106 \\
Std. dev. & 0.031 & 0.032 & 0.261 & 0.194 \\
95\% error & 0.061 & 0.063 & 0.511 & 0.380 \\
RMSE & 0.058 & 0.363 & 0.533 & 0.748 \\
\hline
\end{tabular}

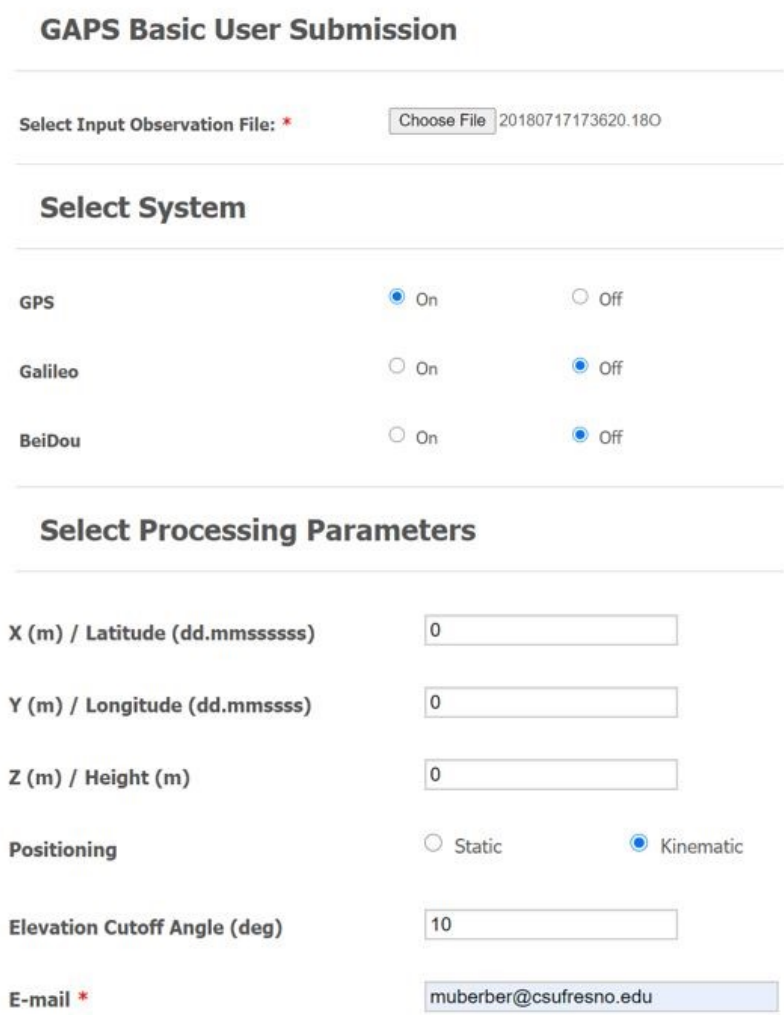

Fig. 7. Data processing parameters used in GAPS.

As can be seen in Table 1, RTKLIB produced the closest results to Agisoft results. Regarding CSRS-PPP results, the mean value of $0.361 \mathrm{~m}$ indicated positive bias. Additionally, RMSE of $0.363 \mathrm{~m}$ implied presence of outliers in the results. GAPS results were not consistent. Moreover, there was an upward shift in the results manifested by the mean value of $0.466 \mathrm{~m}$. Furthermore, standard deviation was the largest among all the software used. In addition, large RMSE implied presence of outliers in the results. Although APPS results were consistent, there was a large negative bias in the results. Nonetheless, this service produced the second largest standard deviation, which made it the least precise service. Further, RMSE for APPS results

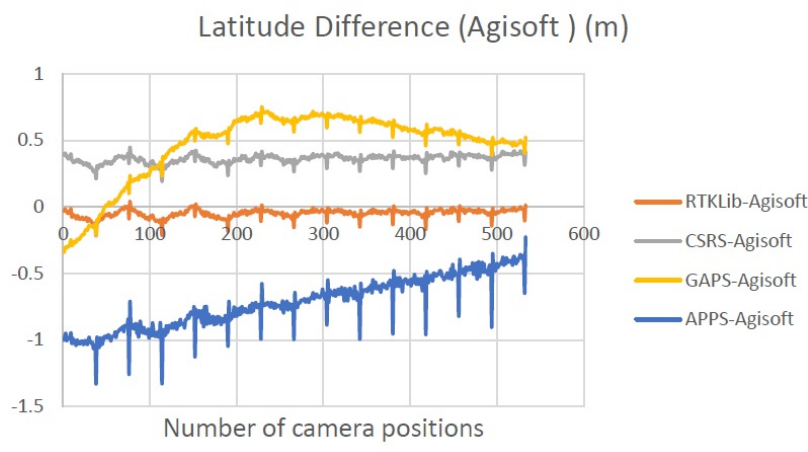

Fig. 8. Latitude differences between camera coordinates that were produced by Agisoft Metashape and the software that was utilized in this project.

was the largest among all the software used, which indicated the lowest accuracy.

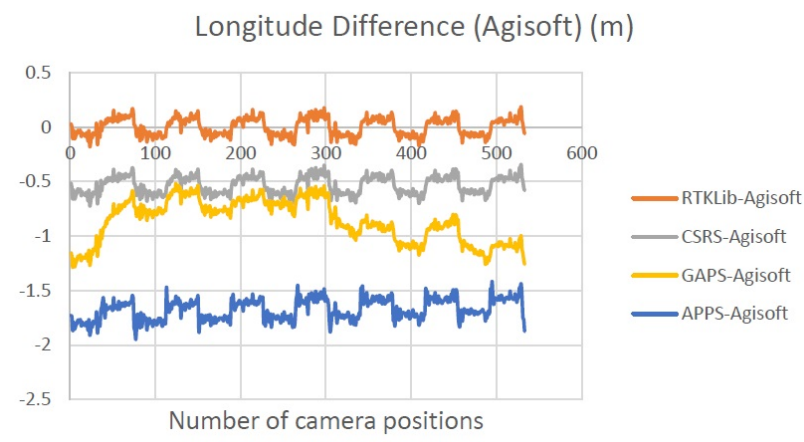

Fig. 9. Longitude differences between the coordinates produced by Agisoft Metashape and the software utilized in this project.

In Table 2, RTKLIB produced the best results in terms of longitude values. Negative bias was present in the CSRSPPP results. Small standard deviation showed high measurement precision. Yet, RMSE of 0.539 hinted at the presence of outliers in the data. GAPS results displayed upward and downward trends. Negative bias was also present in GAPS results. By looking at standard deviation and RMSE values, it was deduced that the results were neither as pre- 
Table 2. Results about longitude differences (Agisoft) (m)

\begin{tabular}{ccccc}
\hline & RTKLIB & CSRS-PPP & GAPS & APPS \\
\hline Min & -0.175 & -0.720 & -1.285 & -1.948 \\
Max & 0.184 & -0.347 & -0.517 & -1.420 \\
Mean & 0.003 & -0.533 & -0.861 & -1.674 \\
Range & 0.359 & 0.373 & 0.768 & 0.528 \\
Std. dev. & 0.081 & 0.081 & 0.195 & 0.098 \\
95\% error & 0.158 & 0.159 & 0.381 & 0.192 \\
RMSE & 0.081 & 0.539 & 0.883 & 1.677 \\
\hline
\end{tabular}

cise nor as accurate as the results produced by RTKLIB and CSRS-PPP. Large negative bias was present in APPS results. Precision of APPS results were $\sim 0.1 \mathrm{~m}$ better than GAPS results. Nevertheless, APPS was the least accurate. These outliers can be ascribed to processing power of the software because although the same data set was submitted to these servers, varying results were obtained. Otherwise, RMSE would be less variable.

Displacements in 2D were calculated using above displayed latitude and longitude results and shown in Fig. 10.

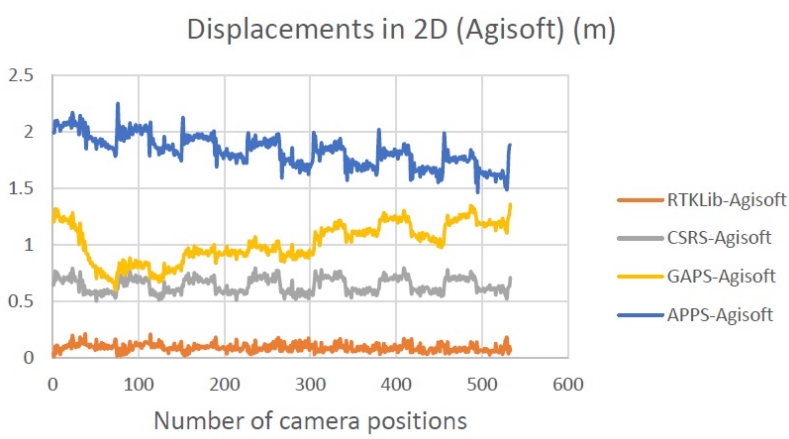

Fig. 10. Displacements in 2D between the coordinates produced by Agisoft Metashape and the software utilized in this project.

Because 2D results were obtained using the latitude and longitude results displayed above, the results in Table 3 follow the results in Tables 1 and 2.

In terms of 1D displacements (Table 4), RTKLIB and CSRS-PPP results were similar i.e., both yielded precision and accuracy in the order of $\mathrm{cm}$ and $\mathrm{dm}$, respectively. There were ups and downs with GAPS results. As such, precision and accuracy of GAPS are in the order of $\mathrm{dm}$. Precision and accuracy of APPS are in the order of $\mathrm{dm}$ and $\mathrm{m}$, respectively.

The preceding comparisons were also made for Pix4Dmapper software and shown in Figs. 12-15.

There are small discrepancies between the results produced by RTKLIB and Pix4D (see Table 5). CSRS-PPP also

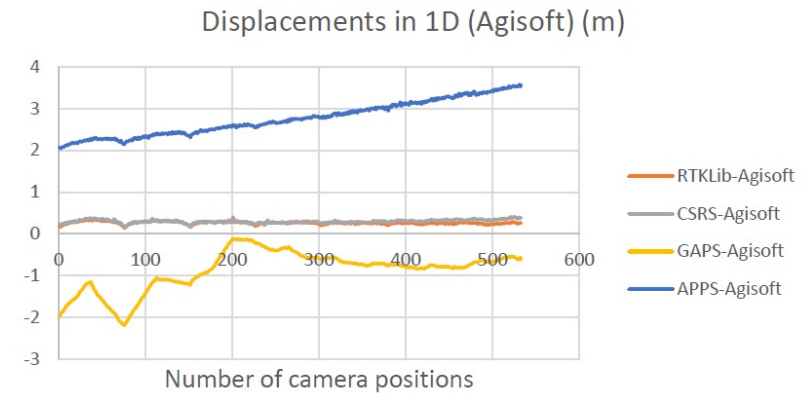

Fig. 11. Displacements in 1D between the coordinates that were produced by Agisoft Metashape and the software utilized in this project.

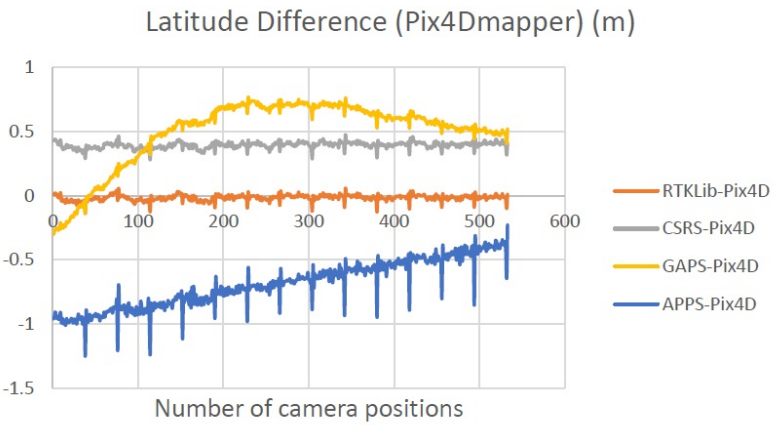

Fig. 12. Latitude differences between the coordinates that were produced by Pix4Dmapper and the software utilized in this project.

produced close results to Pix4D results yet with a positive bias. Large RMSE $(0.395 \mathrm{~m})$ for CSRS-PPP results suggested presence of outliers in the data set. Except in terms of precision $(0.257 \mathrm{~m}$ vs. $0.186 \mathrm{~m})$, GAPS produced better results than APPS. There was a negative bias with APPS results. Largest RMSE of APPS results $(0.713 \mathrm{~m})$ implied that it was the least accurate.

A similar pattern appears in Table 6 as in Table 2 results. RTKLIB produced results closest to Pix4D results. On the other hand, in terms of precision CSRS-PPP results were almost the same as RTKLIB results. Yet, there was a negative bias with CSRS-PPP results. In addition, a large 
Table 3. Results for 2D displacements (Agisoft) (m).

\begin{tabular}{ccccc}
\hline & RTKLIB & CSRS-PPP & GAPS & APPS \\
\hline Min & 0.017 & 0.507 & 0.602 & 1.466 \\
Max & 0.216 & 0.796 & 1.364 & 2.251 \\
Mean & 0.094 & 0.646 & 1.018 & 1.831 \\
Range & 0.200 & 0.289 & 0.761 & 0.785 \\
Std. dev. & 0.033 & 0.066 & 0.171 & 0.137 \\
95\% error & 0.065 & 0.128 & 0.334 & 0.269 \\
RMSE & 0.099 & 0.650 & 1.032 & 1.836 \\
\hline
\end{tabular}

Table 4. Results for 1D displacements (Agisoft) (m).

\begin{tabular}{ccccc}
\hline & RTKLIB & CSRS-PPP & GAPS & APPS \\
\hline Min & 0.141 & 0.168 & -2.192 & 2.049 \\
Max & 0.354 & 0.407 & -0.124 & 3.575 \\
Mean & 0.261 & 0.297 & -0.870 & 2.762 \\
Range & 0.213 & 0.239 & 2.068 & 1.526 \\
Std. dev. & 0.031 & 0.039 & 0.474 & 0.413 \\
95\% error & 0.061 & 0.076 & 0.929 & 0.809 \\
RMSE & 0.263 & 0.300 & 0.991 & 2.793 \\
\hline
\end{tabular}

Table 5. Results about latitude differences (Pix4Dmapper) (m).

\begin{tabular}{ccccc}
\hline & RTKLIB & CSRS-PPP & GAPS & APPS \\
\hline Min & -0.137 & 0.283 & -0.301 & -1.250 \\
Max & 0.057 & 0.474 & 0.767 & -0.225 \\
Mean & -0.016 & 0.394 & 0.499 & -0.689 \\
Range & 0.194 & 0.191 & 1.068 & 1.025 \\
Std. dev. & 0.024 & 0.025 & 0.257 & 0.186 \\
95\% error & 0.046 & 0.048 & 0.503 & 0.364 \\
RMSE & 0.028 & 0.395 & 0.561 & 0.713 \\
\hline
\end{tabular}

Table 6. Results about longitude differences (Pix4Dmapper) (m).

\begin{tabular}{ccccc}
\hline & RTKLIB & CSRS-PPP & GAPS & APPS \\
\hline Min & -0.181 & -0.725 & -1.311 & -1.969 \\
Max & 0.181 & -0.349 & -0.514 & -1.418 \\
Mean & 0.005 & -0.530 & -0.859 & -1.671 \\
Range & 0.362 & 0.376 & 0.797 & 0.551 \\
Std. dev. & 0.080 & 0.081 & 0.193 & 0.099 \\
95\% error & 0.157 & 0.159 & 0.378 & 0.194 \\
RMSE & 0.080 & 0.536 & 0.880 & 1.674 \\
\hline
\end{tabular}

RMSE value points out that CSRS-PPP results were not as accurate as RTKLIB results. Large negative bias also exist in GAPS results. Both precision and accuracy of GAPS results are in the order of $\mathrm{dm}$. There was a large negative bias in APPS results as well. Precision of APPS results is in the order of $\mathrm{cm}$ but its accuracy is in the order of $\mathrm{dm}$.

Displacements in 2D were calculated using above displayed latitude and longitude results (Fig. 14).

A similar pattern appears in Table 7 as in Table 3 results.
As can be seen in Table 8, except small variations, RTKLIB and CSRS-PPP produced similar results. There was a large negative bias with GAPS results and there was a large positive bias with APPS results. In terms of precision, GAPS and APPS results were very close. APPS results were less accurate than GAPS results. 
Table 7. Results for 2D displacements (Pix4Dmapper) (m).

\begin{tabular}{ccccc}
\hline & RTKLIB & CSRS-PPP & GAPS & APPS \\
\hline Min & 0.008 & 0.533 & 0.643 & 1.455 \\
Max & 0.186 & 0.817 & 1.357 & 2.232 \\
Mean & 0.079 & 0.663 & 1.030 & 1.815 \\
Range & 0.178 & 0.284 & 0.714 & 0.778 \\
Std. dev. & 0.031 & 0.064 & 0.166 & 0.135 \\
95\% error & 0.061 & 0.126 & 0.325 & 0.265 \\
RMSE & 0.085 & 0.666 & 1.044 & 1.820 \\
\hline
\end{tabular}

Table 8. Results for 1D displacements (Pix4Dmapper) (m).

\begin{tabular}{ccccc}
\hline & RTKLIB & CSRS-PPP & GAPS & APPS \\
\hline Min & 0.191 & 0.199 & -2.147 & 2.079 \\
Max & 0.271 & 0.381 & -0.134 & 3.555 \\
Mean & 0.239 & 0.275 & -0.892 & 2.740 \\
Range & 0.080 & 0.182 & 2.012 & 1.476 \\
Std. dev. & 0.012 & 0.035 & 0.478 & 0.422 \\
95\% error & 0.024 & 0.068 & 0.937 & 0.826 \\
RMSE & 0.239 & 0.277 & 1.012 & 2.772 \\
\hline
\end{tabular}

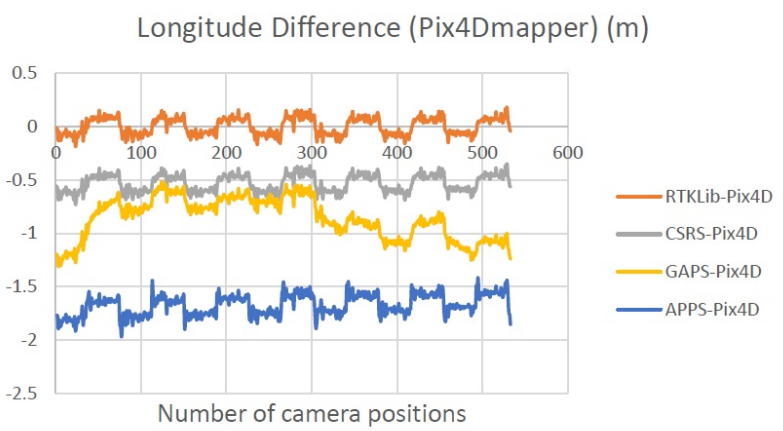

Fig. 13. Longitude differences between the coordinates produced by Pix4Dmapper and the software utilized in this project.

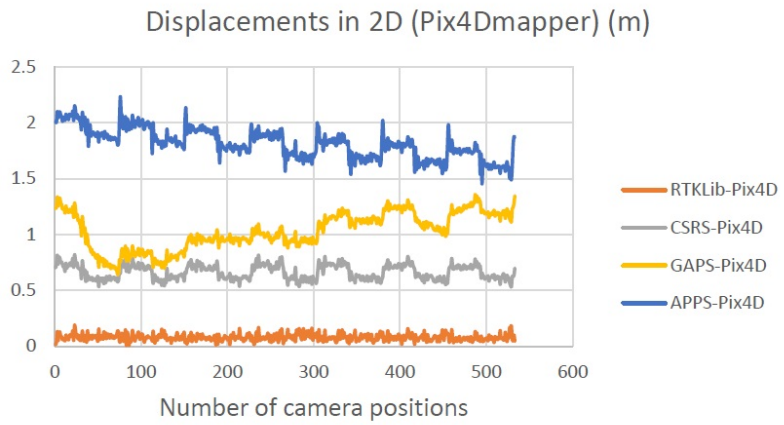

Fig. 14. Displacements in $2 \mathrm{D}$ between the coordinates produced by Pix4Dmapper and the software utilized in this project.

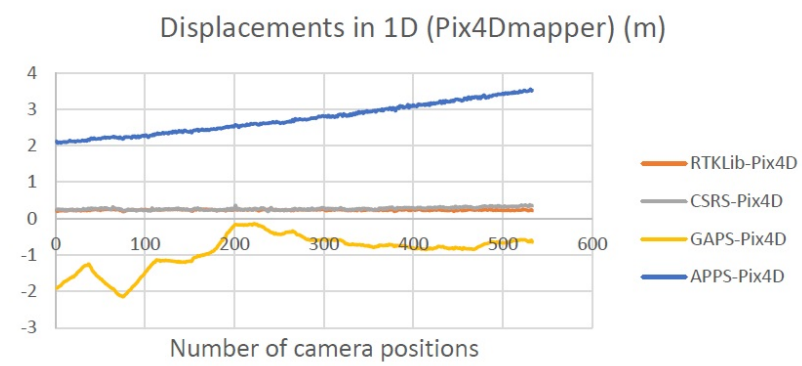

Fig. 15. Displacements in 1D between the coordinates produced by Pix4Dmapper and the software utilized in this project.

\section{Discussions}

It is well established in using online GNSS data processing services that, in static mode, cm-level and, in kinematic mode, $\mathrm{dm}$-level precision is achieved both for horizontal and vertical coordinates. However, with GNSS data analysis, the rule of thumb is that the longer the observation period at a location, the more precise the results will be. In this study, the observation period at a location was a tenth of a second i.e., shorter than the observation period commonly used for kinematic surveys. As such, lower precision is expected from the software used. To summarize precision and accuracy of the software used for both horizontal and vertical coordinates, Table 9 is prepared.

As can be seen in Table 9, despite the fact that tenth of a second data was used in this study, RTKLIB and CSRSPPP achieved cm-level precision. Yet, GAPS and APPS 
Table 9. Precision and accuracy of the software used.

\begin{tabular}{lcccc}
\hline & \multicolumn{2}{c}{ Horizontal Coordinates } & \multicolumn{2}{c}{ Vertical Coordinates } \\
\hline RTKLIB & Precision & Accuracy & Precision & Accuracy \\
CSRS-PPP & $\mathrm{cm}$ & $\mathrm{cm}$ & $\mathrm{cm}$ & $\mathrm{dm}$ \\
GAPS & $\mathrm{cm}$ & $\mathrm{dm}$ & $\mathrm{cm}$ & $\mathrm{dm}$ \\
APPS & $\mathrm{dm}$ & $\mathrm{m}$ & $\mathrm{dm}$ & $\mathrm{m}$ \\
\hline
\end{tabular}

achieved dm-level precision both for horizontal and vertical coordinates.

Cyclic error (up/down pattern) was noticed in horizontal coordinates (latitudinal and longitudinal directions) for the results produced by all the software used. This was due to the time offset between the actual shutter opening and the signal that registered the event. In other words, cyclic error appears due to mid-exposure pulse of the camera timing error. This error is not obvious in the $z$ direction. Cyclic error is not found in literature.

\section{Conclusions}

Lift 1 of the two UAS photogrammetry campaign flown over the San Joaquin Experimental Range resulted in 534 photographs. The executed flight plan was processed using Agisoft Metashape and Pix4Dmapper software. A bundle block adjustment was performed and camera position coordinates were determined by constraining 80 surveyed ground control points and allowing the cameras to freely adjust to their true positions at the instant of exposure. These camera position coordinates coming from Agisoft and Pix4D were considered the baseline and differences between the results produced by these two software and RTKLIB, CSRS-PPP, GAPS, APPS were computed. RTKLIB outperformed the other software that are investigated in this project. This contribution demonstrated the precision and accuracy that should be expected from these software if they are used for kinematic GNSS data processing. The results of this study is limited to the data that were collected during the project day.

\section{References}

Agüera-Vega, F., Carvajal-Ramírez, F., and Martínez-Carricondo, P., 2017, Assessment of Photogrammetric Mapping Accuracy Based on Variation Ground Control Points Number Using Unmanned Aerial Vehicle, Measurement 98, 221-227.
Berber, M., Ustun, A. and Yetkin, M., 2017, Static and kinematic PPP using online services: a case study in Florida, Journal of Spatial Science, DOI: 10.1080/14498596.2017.1292964.

Berber, M. and Wright, W., 2016, Online kinematic GNSS data processing for small hydrographic surveys, Ocean Engineering 112, 335-339.

Berber, M., Ustun, A. and Yetkin, M., 2014, Rapid Static GNSS Data Processing Using Online Services, Journal of Geodetic Science, 4, 123-129.

Chiabrando, F., Lingua, A., and Piras, M., 2013, Direct Photogrammetry Using UAV: Tests and First Results, ISPRS-International Archives of the Photogrammetry, Remote Sensing and Spatial Information Sciences XL-1/W2, 81-86. https://doi.org/10.5194/ isprsarchives-XL-1-W2-81-2013.

Dogru, A. D., Duman, H., Sanli, D. U. and Berber, M., 2018, Efficiency of OPUS-RS solutions, Survey Review, DOI: 10.1080/00396265.2017.1405495

Ebner, R. and Featherstone, W. E., 2008, How well can online GPS PPP post-processing services be used to establish geodetic survey control networks?, Journal of Applied Geodesy, 2, 149157.

Fraser, C. S., 1997, Digital camera self-calibration, ISPRS Journal of Photogrammetry \& Remote Sensing 52 (1997) 149-159.

Ghilani, C. and Wolf, P. R., 2007, Elementary Surveying - An Introduction to Geomatics, 12th edition, Pearson Prentice Hall, New Jersey.

Ghoddousi-Fard, R. and Dare, P., 2006, Online GPS processing services: An initial study, GPS Solutions, 10, 12-20.

Jamieson, M. and Gillins, D. T., 2018, Comparative Analysis of Online Static GNSS Postprocessing Services, Journal of Surveying Engineering, vol. 144, issue 4

Kemper, G., Melykuti, B. and Yu, C., 2016, Calibration Procedures on Oblique Camera Setups, The International Archives of the Photogrammetry, Remote Sensing and Spatial Information Sciences, Volume XLI-B1, 2016 XIII ISPRS Congress, 12-19 July 2016, Prague, Czech Republic.

Liu, J-H. and Shih, T-Y., 2007, A performance evaluation of the internet based static GPS computation services, Survey Review, 39, 304, 166-175.

Nocerino, E., Menna, F., Remondino, F. and Saleric, R., 2013, Accuracy and Block Deformation Analysis in Automatic UAV and Terrestrial Photogrammetry - Lesson Learnt, ISPRS Annals of the Photogrammetry, Remote Sensing and Spatial Information Sciences, Volume II-5/W1, 2013 XXIV International CIPA Symposium, 2 - 6 September 2013, Strasbourg, France.

Pérez, M., Agüera, F. and Carvajal, F., 2011, Digital Camera Calibration using Images Taken from an Unmanned Aerial Vehicle, International Archives of the Photogrammetry, Remote Sensing and Spatial Information Sciences, Vol. XXXVIII-1/C22 UAV-g 
2011, Conference on Unmanned Aerial Vehicle in Geomatics, Zurich, Switzerland.

Snay, R., Choi, K., Gerald, M., Schwarz, C., Soler, T. and Weston, N., 2011a, How precise is OPUS? Part I: Experimental results, The American Surveyor, vol. 8, no. 5.

Snay, R., Choi, K., Gerald, M., Schwarz, C., Soler, T. and Weston, N., 2011b, How precise is OPUS? Part II: Available precision estimates, The American Surveyor, vol. 8, no. 6.

Snay, R., Choi, K., Gerald, M., Schwarz, C., Soler, T. and Weston, N., 2011c, How precise is OPUS? Part III: The rest of the story, The American Surveyor, vol. 8, no. 7.

Soler, T., Michalak, P., Weston, N. D., Snay, R. A. and Foote, R. H., 2006, Accuracy of OPUS solutions for 1 to $4 \mathrm{~h}$ observing sessions, GPS Solutions, 10, 45-55.

Tekavec, J., Oštir, K., Lisec, A. and Štebe, G., 2019, A System for Monitoring of UAV Camera Orientation: Design and Initial Analysis, ISPRS Annals of the Photogrammetry, Remote Sensing and Spatial Information Sciences, Volume IV-2/W5, 2019 ISPRS Geospatial Week 2019, 10-14 June 2019, Enschede, The Netherlands.

Tsakiri, M., 2008, GPS Processing Using Online Services, Journal of Surveying Engineering, vol. 134, no. 4, 115-125. 